\title{
A Standardized Protocol for Radical Mastectomy Specimens at Hospital Universitario Antonio Pedro - Niteroi - Brazil: An Anatomopathological Perspective
}

\author{
Fabiana Resende Rodrigues* Wilhermo Torres and Mayra Carrijo Rochael \\ Pathology Service, Antonio Pedro Universitary Hospital (HUAP), Federal Fluminense University (UFF), Niterói, Rio de Janeiro, Brazil
}

\begin{abstract}
Introduction: Breast cancer is the second most common cancer worldwide. Adequate evaluation of the specimen is essential for accurate diagnosis and prognosis.

Objective: To present a standardized protocol for the gross and microscopic examination of radical mastectomy specimens, despite the presence or absence of homolateral axillary dissection and irrespective of the type of the malignancy to provide the necessary information to guide potential adjuvant therapy and prognostic information.

Materials and methods: A standardized protocol was created for the examination of surgical specimens from radical mastectomies.

Results: The group of pathologists responsible for routine examination agreed that use of the standardized protocol reduced the time spent on macroscopic and microscopic examination of the radical mastectomy specimens and increased the accuracy of recording the information needed for diagnosis.

Conclusion: We believe that simplification of the protocol presented here for guidance of histological examination of surgical mastectomy specimens is easily applied, rapid, reliable and safe, and will contribute to the routine diagnostic practice.
\end{abstract}

\section{Introduction}

Breast cancer is the second most common cancer in the world, after lung cancer (when not considering skin cancer) and occurs more frequently in women than in men [1]. Each year, approximately $20 \%$ of new cancers in women are breast cancers [2]. The approach to treat breast lesions has undergone significant modifications, evolving from radical to more economical surgeries, with the aim of reducing morbidity as well as improving aesthetics and the quality of life. The correct evaluation of neoplasias, surgical margins, the presence of neoplastic lymphatic and/or blood vessels emboli, perineural neoplastic invasion, microcalcifications, necrosis and metastasis to local lymph nodes, neoplastic extra-capsular lymph node invasion, extra-capsular extension and coalescence of positive lymph nodes become essential factors for prognostic evaluation and the success of clinical oncological treatment. The ideal strategy in approaching these specimens is provided in the anatomopathological report, required for diagnosis and appropriate, reliable and efficient treatment. Here we developed a standardized version of this specific protocol, with reference to recommendations by the Association of Directors of Anatomic and Surgical Pathology (ADASP) and the College of American Pathologists (CAP) $[3,4]$.

\section{Objectives}

To propose a standardized protocol for gross and microscopic examination of radical mastectomy specimens, with or without homolateral axillary dissection and irrespective for the type of malignant neoplasia. The protocol is based on a rapid response checklist containing items including evaluation of the demographic and/or clinical data.

\section{Materials and Methods}

A standardized protocol for the macroscopic and microscopic examination of radical mastectomy specimens was prepared by a committee of pathologists, surgeons, oncologists, based on ADASP and CAP protocols [3-4]. This protocol refers only to post-operative examination. A logistic scheme for the histopathological analysis of the mastectomy specimen can be seen in Scheme 1.

Data elements were presented as a standardized checklist, with one page for macroscopic examination and one page for microscopic examination, where each data element corresponds to a gap that must be completed by the examiner and spaces to be filled with weights and measurements (Table 1).

All surgical material was previously fixed in $10 \%$ buffered formalin. This study included cases of mastectomy with axillary lymph node dissections, as proposed by Halsted and modified by Patey. The method for macroscopic examination was as follows: the surgical specimen was placed on the exam table (or a washable bench), the anterior region was examined first (skin up), the laterality of the specimen, the presence of the pectoral minor muscle and the presence of the homolateral axillary dissection were observed and confirmed (the presence of the axillary dissection aids the anatomical positioning of the specimen).

*Corresponding author: Fabiana Resende Rodrigues, MD, Pathology Service Antonio Pedro Universitary Hospital (HUAP), Federal Fluminense University (UFF), Niterói, Rio de Janeiro, Brazil, E-mail: resendefr@yahoo.com.br

Received February 08, 2012; Accepted March 28, 2012; Published March 30, 2012

Citation: Rodrigues FR, Torres W, Rochael MC (2012) A Standardized Protoco for Radical Mastectomy Specimens at Hospital Universitário Antonio Pedro Niterói - Brazil: An Anatomopathological Perspective. J Cancer Sci Ther 4: 056060. doi:10.4172/1948-5956.1000111

Copyright: (c) 2012 Rodrigues FR, et al. This is an open-access article distributed under the terms of the Creative Commons Attribution License, which permits unrestricted use, distribution, and reproduction in any medium, provided the original author and source are credited. 
To assist in positioning the surgical specimen some markings has been made intra-operatively or post-operatively with sutures or wire by the surgeon. The markings adopted by our service, in accordance with the surgical staff and used in this protocol are referred to by the mnemonic "SIM", where the "S" corresponds to the superior margin marked with one suture or wire, "I" corresponds to the inferior margin marked with two sutures or wires and " $\mathrm{M}$ " to the medial margin, identified by three sutures or wires. In all cases the breast was palpated for identification of the tumor location, in correlation with clinical data provided by the requesting physician or assistant (Figure 1).

The excised specimens were weighed $(\mathrm{g})$ and measured $(\mathrm{cm})$. The pectoral minor muscle and axillary content were weighed together with the breast and then measured separately. The cutaneous layer was measured and the presence, location and measurements of recent or old scarring were reported. The presence of the nipple, its location, shape and measurements were reported, as well as the presence of other lesions, retractions or ulcers on the skin.

The posterior or deep region of the surgical specimen was examined and dried with a paper towel and then the lateral and deep margins were inked along with the axillary dissection. Once the ink had dried, the specimen was oriented anatomically and the anterior portion was placed against the cutting board as if the dissector were

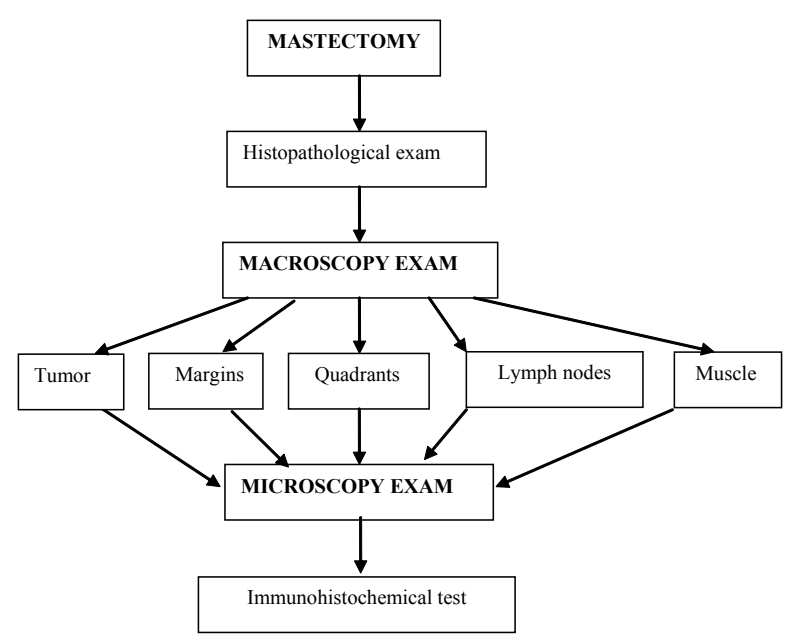

Scheme 1: A logistic scheme of the analysis of histopathological exam of mastectomy.

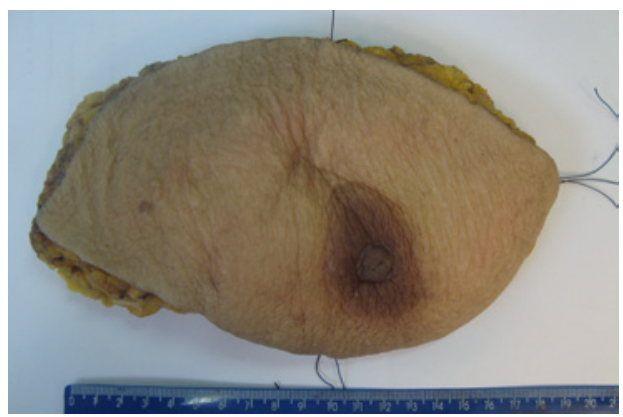

Figure 1: Gross examination of cancer breast: Right Breast - previous view: the mnemonic "SIM".

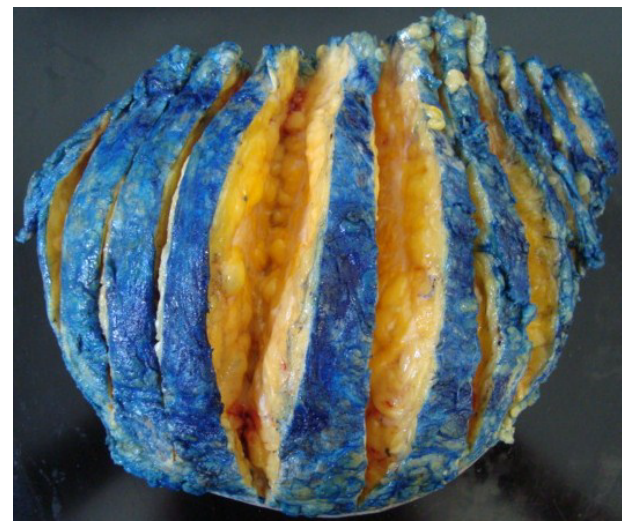

Figure 2: Gross examination of cancer breast: Right Breast - back view with of blue paint and cut.

seeing it from the posterior aspect. A sharp knife was used to slice the specimen and the cut was extended up to the skin without sectioning it. In order to find small tumoral foci and/or multifocality and/or multicentricity, slicing the specimen to the thinnest size possible is always recommended, ideally to a width of $0.5 \mathrm{~cm}$ (Figure 2).

The sampling was included in histological cassettes where slices were cut to an average thickness of $0.2 \mathrm{~cm}$. The sampling depended on the complexity of the specimen.

\section{Tumor}

Surgical cavity or tumors were identified from the histopathological specimens and its topography was reported. For this, we followed the nomenclature of a report of the SISMAMA program by the Ministry of Health - INCA (2011) which refers to the quadrants: UQlat = Union of lateral quadrants, UQInf = Union of inferior quadrants; UQsup = Union of superior quadrants; UQM = Union of medial quadrants; QSL = superior lateral quadrant, QSM = superior medial quadrant; QIL = inferior lateral quadrant; QIM = inferior medial quadrant and RRA = retroareolar region [5]. Where a surgical cavity was present we measured and described the walls and in the case of a tumor we measured and described the color, consistency, contours and boundaries. We reported the presence of multifocality (more than one tumor in the same quadrant or at a distance of $3 \mathrm{~cm}$ from the main lesion) and multicentricity (a tumor in more than one quadrant at a distance of more than $3 \mathrm{~cm}$ from the main lesion). For the cleavage, we made sections of $0.4 \mathrm{~cm}$ and the smallest possible representation for the six blocks. This included more fragments when the morphological aspect required greater representation. We chose to always include the periphery of the tumor, where there are a greater number of neoplastic lymphatic emboli and/or blood vessels and neoplastic perineural invasion. However, at least one block was of the central region of the tumor to represent the area of possible tumor necrosis.

\section{Margins}

During resection the surgeon has to identify the margins and we recorded the distance between the limits of the tumor or the cavity of a previously removed tumor to the closest margin(s) and to the overlying skin. We used six blocks, representing the skin/nipple and the superior, inferior, medial/internal, lateral/external and deep/muscular margins. All tumor prone areas should be submitted for histologic processing. 
Citation: Rodrigues FR, Torres W, Rochael MC (2012) A Standardized Protocol for Radical Mastectomy Specimens at Hospital Universitário Antonio Pedro - Niterói - Brazil: An Anatomopathological Perspective. J Cancer Sci Ther 4: 056-060. doi:10.4172/1948-5956.1000111

\section{GROSS:}

\section{Breast- laterality:}

weight \& size:

Markings intra-operatively:

Cutaneous flap:

Surgical incision:

Other skin lesions:

Nipple:

Surgical cavity sections, location:

Tumor:

Local:

Color:

Consistency:

Contours:

Margins:

Measure and distance:

Multicentricity in:

Surrounding parenchyma:

Axillary adipose tissue:

Lymph nodes and number:

Conglomerates:

Small pectoral muscle:

MICROSCOPIC:

Carcinoma type:

In situ or infiltration:

Final histological grade:

Measures:

Location and multifocality:

Invasion of skin:

Paget disease:

Lymphatic emboli:

Blood vessel emboli:

Perineural invasion:

Microcalcifications:

Necrosis:

Lymphoplasmocitaric infiltrate:

Areas of in situ carcinoma:

Multicentricity:

Other quadrants:

Surgical margins:

Lymph nodes:

Small pectoral muscle:

Immunohistochemistry test

Oestrogen receptor

Progesterone receptor

Cerb-B2/ Her2

pTNM

Signature
( ) right ( ) left ( ) not specified

weight _ $\mathrm{g} ; \ldots \mathrm{x} \_\mathrm{x} \_\mathrm{cm}$

( ) with ( ) at 12 hrs ( ) "SIM" type ( ) without markings

of __ $\mathrm{x} \_\mathrm{cm}$

( ) with ( ) without ( ) recent ( ) old, of __ _ _cm

( ) retraction ( ) ulceration ( ) lesion:___ which measures_x_cm, a distance of _cm from the nipple

( ) protrusive ( ) flat ( ) retracted, ( ) with lesion ( ) without lesion and measures_x_cm

( ) absent ( ) present, measures_x_cm, localised_, filled ( ) by clots ( ) gauze, with walls ( ) soft ( ) white ( ) hardened

( ) absent ( ) single ( ) multiple ( ) residual ( ) multifocality

( ) UQlat ( ) UQInf ( ) UQsup ( ) UQmed ( ) QSL ( ) QSM ( ) QIL ( ) QIM ( ) RRA

( ) white-ish ( ) yellow-ish ( ) reddish ( ), with dots ( ) yellow-ish ( ) reddish

( ) firm ( ) soft ( ) elastic ( ) friable

( ) spiculated ( ) lobulated

( ) imprecise ( ) precise

of _ $\mathrm{X} \_\mathrm{cm}$, distance_cm from the closet surgical margin ( ) superior ( ) inferior ( ) medial ( ) lateral ( ) deep

( ) UQlat ( ) UQInf ( ) UQsup ( ) UQmed ( ) QSL ( ) QSM ( ) QIL ( ) QIM ( ) RRA, a distance of_cm from the main lesion

( ) yellowed or untuous, with ( ) whitened and elastic areas, ( ) cysts ( ) and separated by trabeculae measures_X_X_cm

( ) none ( ) isolated _ (number), the greatest measure _ $\mathrm{x} \_\mathrm{cm}$, measure $\mathrm{x}_{-} \mathrm{x} \_\mathrm{cm}$

measure $x \times \mathrm{cm}$

( ) Absent ( ) Present

measures $\mathrm{x} \times \mathrm{x} \_\mathrm{cm}$, brownish and fasciculated

( ) ductal ( ) lobular ( ) mixed ( ) medular ( ) other:

( ) in situ ( ) infiltrate ( ) residua

( ) affected by autolysis, (1-3) by Nottingham modified by ELSTON \& ELLIS (1991) (tubular formation: $(1=>75 \%$, 2=10-75\%; 3=>75\%); atypia:_(1=small, 2=moderate; $3=$ extensive); mitoses:_ $(1=<7 / 10 \mathrm{hpf}, 2=10 / 15 \mathrm{hpf} ; 3=>15 \mathrm{hpf})$ measures_cm at the greatest axis

( ) UQlat ( ) UQInf ( ) UQsup ( ) UQmed ( ) QSL ( ) QSM ( ) QIL ( ) QIM ( ) RRA ( ) multifocal

( ) Absent ( ) Present, ( ) including the dermis

( ) Absent ( ) Present

( ) Absent ( ) Present

( ) Absent ( ) Present

( ) Absent ( ) Present

( ) Absent ( ) Present

( ) Absent ( ) Present

( ) Absent ( ) Present ( ) mild ( ) moderate ( ) severe

( ) Absent ( ) Present ( ) mild ( ) moderate ( ) severe

( ) Absent ( ) Present in the main lesion ( ) Present outside the main lesion at a ( ) low ( ) intermediate ( ) high degree

( ) Absent ( ) Present

( ) Fibrocystic mastopathy ( ) adenosis ( ) apocrine metaplasia ( ) epithelial hyperplasia ( ) others:

( ) free ( ) compromised ( ) distance $\mathrm{cm}$ from the closest surgical margin ( ) superior ( ) inferior ( ) medial ( ) lateral ( ) deep

( ) free of neoplasia ( ) $\left(\mathrm{N}^{\circ}\right)$ of compromised neoplastic lymph nodes between $\left(\mathrm{N}^{\circ}\right)$ isolates, ( ) with neoplastic capsular invasion ( ) extracapsular extension to extranodal tissue ( ) neoplastic lymphatic emboli ( ) blood vessels. The greatest metastasic focus_cm in diameter

( ) free ( ) compromised

After microscopic analysis (preferably of the histological block most representative of the infiltrate tumor)

( ) $0 \%$ ( ) $1-10 \%$ ( ) $10-25 \%$ ( ) $25-50 \%$ ( ) $50-75 \%$ ( ) $>75 \%$

( ) $0 \%$ ( ) $1-10 \%$ ( ) $10-25 \%$ ( ) $25-50 \%$ ( ) $50-75 \%$ ( ) $>75 \%$

( ) 0 ( ) 1 ( ) 2 ( ) 3 ( )

pT $\quad \mathrm{N}$


The orientation of the fragments representing the margins should be taken parallel to the cut surface unless the distance between the boundaries of the tumor and the margin are less than $2.0 \mathrm{~cm}$.

\section{Other quadrants}

We described all other lesions other than the tumor if present. We used a minimal representation of one block per quadrant.

\section{Lymph nodes}

Dissection of the adipose tissue was performed using an instrument to cut fine, parallel slices of about $4 \mathrm{~mm}$. Lymph nodes greater than $4 \mathrm{~mm}$ were visually identified while lymph nodes smaller than $4 \mathrm{~mm}$ were detected by tactile perception. All lymph nodes in their entireties should be submitted for histological examination. Those measuring 0.4 $\mathrm{cm}$ or less do not need to be sectioned like the larger ones; which are cut as many times as necessary so as to provide $0.4 \mathrm{~cm}$ thick fragments. The remaining adipose tissue was stored for reference.

\section{Muscle}

Muscle at the deep margin should be measured and represented histologically. If there is tumor in this area it should submitted accordingly to evaluate the amount of negative tissue between the neoplasia and the surgical line of resection.

The method for microscopic examination of surgical specimens was as follows:

\section{Tumor}

The histological type was determined using the International Classification of Tumors of the World Health Organization (WHO), and the histological degree/grade determined by Nottingham modified by Elston and Ellis [6]. Microscopic evaluation was applied to viable areas with a high concentration of tumor and a high mitotic index. In the microscopic report we noted the greatest diameter of the tumor, the location, neoplastic invasion of the overlying skin, neoplastic lymphatic emboli and or blood vessels, perineural invasion, microcalcifications, necrosis, the quantity of lymphocytic infiltrate, areas of carcinoma in situ outside the main lesion and its grade and the presence of multifocal or multicentric breast carcinoma. Immunohistochemical analysis for oestrogen and progesterone receptors and Cerb-B2 was requested for all cases in which these markers were not studied in the previous biopsies.

\section{Margins}

The inking allowed a precise evaluation of the distance from the tumor to the surgical margins by microscopy. In the case of positive margins, the pathologist should report if the carcinoma is in situ or invasive and the extent of the involvement and whether focal or diffuse. We consider a negative margin when the distance between the tumor and the margin is greater than one centimetre. Margins will be considered positive if there is $0.5 \mathrm{~cm}$ or less of healthy tissue between the tumor and the resection line. Safety margins with $0.5 \mathrm{~cm}$ or less should be reported to the surgeon for him to decide if the they should be enlarged or not.

\section{Other quadrants}

Other neoplastic conditions were described if present.

\section{Lymph nodes}

We reported the size of the largest metastatic focus, neoplastic invasion of the capsule, extracapsular extension, lymphatic neoplastic emboli and/or blood vessels, neoplastic perineural invasion, calcifications, necrosis and coalescence of positive lymph nodes. Microscopic examination of all lymph nodes in their entireties should be performed.

Each histological block was specified according to the area and the number of fragments. They were fixed in $10 \%$ buffered formaldehyde and subsequently embedded in paraffin at $58^{\circ} \mathrm{C}$ for automated routine histological processing. From the paraffin block, $4 \mu \mathrm{m}$ histological sections were made by a microtome, stretched in a water bath and fixed on glass slides.

The macroscopic findings were documented in 7.2 megapixels Sony DSC-W125 Effective Zeiss camera.

\section{Results}

This protocol for surgical specimens was performed in a university hospital with the guidance of medical pathology residents with a mean time reduction of around 1 to $2 \mathrm{hrs}$. The reason for time reduction is based mainly on the filling-in of a "yes/no" form for both gross and microscopic descriptions. Time was also reduced due to the standardization of the number of blocks from 20 to 25 cassettes. The average number of cassettes was 20 to 25 cassettes/ case, including about six cassettes for the tumor, 1 cassette for the skin/nipple, one cassette for each non neoplastic quadrant, one cassette for each surgical margin, and 5 to 10 cassettes for the lymph nodes.

\section{Discussion}

Anatomopathological and cytopathological examination are becoming increasingly more integrated into the practice of mastology. The information contained in the completed report is not only of diagnostic value but provides guidelines for the monitoring and treatment of patients with breast disease, particularly cancer. TNM staging is an important survival factor and a measurement of disease severity. Staging is also essential for cancer comparative studies. Familiarity with the histological nomenclature of lesions, most common in surgical diagnostic practice is important when dealing with breast disease [7].

In this study we emphasize a standardized methodology to report the examination of mastectomy surgical specimens. We aim to guide pathologists and standardize the approach for surgical specimen examination, providing useful information for clinical oncologists and avoiding the omission of relevant gross and microscopic data. The development of specific protocols for the systematic study of surgical specimens has been a concern of specialised services as well as of pathology associations such as ADASP and CAP [3-4]. The protocols provide general guidelines regarding macroscopy, sectioning, suggesting the amount of tissue and block sampling and microscopy. This protocol is based on protocols already established by specialised literature such as a study by Xavier et al. [8]. The latter shows a specific protocol for the segmenting of impalpable breast lesions. Based on this and due to the fact that a lot of essential and accurate information is often missing from histopathological reports for the oncological treatment of patients with breast cancer, we created a standardized protocol for radical mastectomies, appropriate to the routine procedure 
in our laboratory. The protocols are frequently reassessed and should be updated whenever necessary.

The physician or surgeon requesting examinations are coresponsible for providing clinical data for the histological requisition which is of utmost importance, as well as the correct identification of where the specimen will be sent and also the way the specimen is to be sent. The recommended quantity of formaldehyde is always ten times the volume of the surgical specimen.

The presence of axillary content together with the surgical specimen from mastectomy helps in positioning the specimen, mainly when not identified by the surgeon. Absent or inadequate anatomical markings make it impossible to provide accurate and reliable information about the tumor and the surgical margins [9]. The marking of the breast can be analogous to a watch (12 O'clock), which is the most common form of marking. It can also be marked by the "SIM" mnemonic reported above. Regardless of the marking method chosen, it is important that the surgeon opts for one of these methods and informs the pathologist in which form the specimen is marked.

Methodologically, macroscopic and microscopic examination of the anatomical specimens should be performed in a segmented and ordered manner, so as not to omit relevant data. Therefore the following points should be considered: 1) Measurements of surgical specimens should always be taken in three dimensions with the objective to impute the volume; 2) Marking the surgical margins of the specimen - it is extremely important to mark with India ink or similar as it is technically difficult to identify the true margin when this is not performed. There is consensus in the literature that a tumor present on any inked surface represents a positive margin. However, the definition of a negative margin is not uniform [10]. Some authors propose a distance that can vary by one, two or five millimetres between the tumor and the margin for it to be considered negative [11]. Others consider a negative margin any distance between the tumor and the margin, even if it is represented by a few cells or fibres [12]. A positive margin does not necessarily mean that there is a residual breast tumor. However the risk of residual disease is high if the involvement of margins is compromised $[13,14]$. Similarly, negative margins do not always guarantee the absence of residual disease or local recurrence [9].

The time between the extraction of the specimen, adequate $10 \%$ buffered formalin fixation, processing and immunohistochemical testing are important factors that interfere with autolysis of the material and diagnosis. The amount of time between surgical removal and immersion in formalin solution, as well as the time the specimen remained in the fixation before the initiation of histological processing are important factors that interfere with immunohistochemistry results. Ideally time spent in formalin should not exceed 72 hours and should not be less than six hours. The interval between extraction of specimen and immersion in formalin should be as fast as possible. Immunohistochemistry to test for markers of breast tumours should be performed on the histological block which is most representative of the infiltrate tumor and with little or no necrosis. The expression of hormonal receptors, oestrogen and progesterone, are associated with a neoplasia of low histological grade and with better anti-estrogenic treatment response. Cerb-B2 is an oncogene presented in the literature with various names and different spellings: c-erbB-2, cerbB-2, C-erbB-2, Her2, Her-2/neu, ERBB2, erbB2, erbB-2, neu/ c-erbB-2, oncogene neu, protein neu and neu. The location of this receptor on the cell membrane makes it an ideal target molecule for adjuvant or neoadjuvant therapy. Its over expression is related to breast tumours with aggressive biological behaviour, mainly in patients with positive lymph nodes or may be associated with worse disease prognosis, reduced survival time, rapid growth of the tumor and a change in the frequency of recurrence after surgical intervention [4].

Internal quality control in laboratories of pathological anatomy is always necessary especially when there is discordance between the clinical report and the patient report issued. When a discrepancy occurs, the re-examination of the surgical specimen becomes imperative.

\section{Conclusion}

There was consensus by the group of pathologists responsible for routine work that the use of the standardized protocol checklist reduced the time spent on macroscopic and microscopic examinations of radical mastectomy specimens and increased the accuracy of recording the information needed for diagnosis.

In conclusion we believe that the standardized protocol presented here is easy to use, and brings security and confidence in making a diagnosis. We hope that this standardized protocol will be used by other pathologists and that clinical oncologists will have knowledge of all steps of anatomopathological examination.

\section{References}

1. (2009) WHO (World Health Organization).

2. (2008) IARC (International Agency for Research on Cancer). In: World Cancer Report.

3. (2004) Recommendations For The Reporting Of Breast Carcinoma.

4. (2011) CAP (College of American Pathologists).

5. (2011) Ministry of Health - Rio de Janeiro RJ.

6. Elston CW, Ellis IO (1991) Pathological prognostic factors in breast cancer. I. The value of histological grade in breast cancer: experience from a large study with long-term follow-up. Histopathology 19: 403-410.

7. (2011) AJCC (American Joint Committee on Cancer).

8. Xavier, GR, Vilasboas SA, Scaldaferri MA, Gobbi H (2005) Carcinomas não palpáveis de mama: avaliação e experiência com protocolo específico para estudo anatomopatológico. J Bras Patol Med Lab 41: 51-60.

9. Rosen PR (2009) Pathology examination of breast specimes. In Rosen's Breast Pathology. Ed Wolters Kluwer/Lippincott Willians \& Wilkins, Philadelphia.

10. Schnitt SJ, Abner A, Gelman R, Connolly JL, Recht A, et al. (1994) The relationship between microscopic margins of resection and the risk of local recurrence in patients with breast cancer treated with breast-conserving surgery and radiation therapy. Cancer 74: 1746-1751.

11. Freedman G, Fowble B, Hanlon A, Nicolaou N, Fein D, et al. (1999) Patients with early stage invasive cancer with close or positive margins treated with conservative surgery and radiation have an increased risk of breast recurrence that is delayed by adjuvant systemic therapy. Int J Radiat Oncol Biol Phys 44 1005-1015.

12. Singletary SE (2002) Surgical margins in patients with early-stage breast cancer treated with breast conservation therapy. Am J Surg 184: 383-393.

13. Gennaro M, Ferraris C, Guida V, Tomasic G, Carcangiu ML, et al. (2001) Conservative surgery in breast cancer. Significance of resection margins. Breast 10: 432-437.

14. Silverstein MJ, Lagios MD, Groshen S, Waisman JR, Lewinsky BS, et al (1999) The influence of margin width on local control of ductal carcinoma in situ of the breast. N Engl J Med 340: 1455-1461. 